\title{
Comparison of Three Prediction Rules for Assessing the Risk of Gastric Cancer in Chinese Health Examination Populations
}

\section{Xiaolao Xie}

Shanghai Songjiang Clinical medical college of Nanjing Medical College

\section{Yuexing Lai}

Songjiang Hospital Affiliated to Shanghai Jiaotong Univerity School of Medicine

\section{Ping Xu ( $\sim$ sjzxxp@yeah.net)}

Songjiang Hospital Affiliated First People's Hospital, Shanghai Jiao Tong University https://orcid.org/0000-0003-0270-9841

\section{Kai Xu}

Songjiang Hospital Affiliated to Shanghai Jiaotong University School of Medicine

\section{Jing Wang}

Songjiang Hospital Affiliated to Shanghai Jiaotong University School of Medicine

\section{Libo Wang}

Songjiang Hospital Affiliated to Shanghai Jiaotong University School of Medicine

\section{Research article}

Keywords: Gastric cancer, Screening, Early diagnosis, Gastroscopy

Posted Date: February 25th, 2021

DOI: https://doi.org/10.21203/rs.3.rs-257833/v1

License: (ㅇ) (i) This work is licensed under a Creative Commons Attribution 4.0 International License. Read Full License 


\section{Abstract}

Background The current consensus regarding gastric cancer screening in China recommends Li's Scoring System to assess the risk of gastric cancer. Objectives To compare the predictive capacity of three prediction rules: the ABC method, the Scoring System from the Japan Public Health Center (JPHC), and Li's Scoring System in Chinese health examination populations.

Methods We retrospectively evaluated 1,436 patients undergoing gastroscopy. The patients were classified into three groups (low, medium and high risk) according to each rule. The predictive capacity of three rules to assess the risk of gastric cancer was compared.

Results A total of 28 (1.95\%) cases with gastric cancer were detected. The Scoring System from JPHC and Li's Scoring System performed similarly, and the areas under the receiver-operating characteristic (ROC) curves (AUC) were 0.745 (95\% Cl: $0.722-0.767)$, and 0.739 (95\% Cl: $0.715-0.761)$, respectively. And the AUC for the ABC method was 0.642 (95\%Cl: 0.617-0.667), significantly lower than that for the Scoring System from JPHC $(p<0.05)$.Li's Scoring System had the highest sensitivity, significantly higher than that of the Scoring System from JPHC ( $85.71 \%$ vs $53.57 \%, p<0.05)$. Larger proportions of low-risk patients were diagnosed as gastric cancer by the Scoring System from JPHC (1.99\%) and ABC method $(0.99 \%)$ than by Li's Scoring System $(0.55 \%)$.

Conclusions The Scoring System from JPHC and Li's Scoring System have a similar performance in assessing the risk of gastric cancer, but Li's Scoring System is more effective in Chinese health examination populations because of the lowest probability of missed diagnosis.

\section{Background}

Gastric cancer is a common malignant tumor in China. In 2015, an estimated 679,100 Chinese were newly diagnosed with gastric cancer, and 498,000 people in China ultimately died from the disease, accounting for approximately $50 \%$ of new cases and deaths worldwide [1]. The prognosis for gastric cancer is closely related to the stage of the disease at the time of diagnosis [2-4]. At present, the detection rate of early gastric cancer in China is less than $10 \%$, far less than in Korea and Japan, where national cancer screening programs have been launched [5-9].

The current screening guidelines for gastric cancer in China suggest that beginning gastric cancer screening at the age of 40 for all "high risk" populations: viz., those with Helicobacter pylori (H. pylori) infections; a positive family history of gastric cancer; precancerous diseases of gastric cancer such as chronic atrophic gastritis, gastric ulcers, and gastric polyps; risk factors for gastric cancer such as smoking, heavy alcohol drinking, and high salt diets; or those residing in high incidence regions for more than 3 years [10]. Upper gastrointestinal endoscopies in combination with mucosa biopsies for histological examinations are currently regarded as the gold standard for diagnosing gastric cancer. However, "high risk" populations are estimated to exceed 300 million people in China [11]. It is difficult to carry out a mass screening program for entire populations, owing to high costs and insufficient 
endoscopists and equipment. Consequently, the current status of diagnosis and treatment of gastric cancer can be improved by stratifying the risk of gastric cancer and identifying individuals with a high risk for gastroscopies.

To characterize the risk of gastric cancer, a number of prediction rules that incorporate multiple risk factors have been developed. Miki developed the ABC method (Table 1), which combines H. pylori serology with pepsinogen (PG) level, and screened individuals were classified into four groups based on the results: Group A, Group B, Group C, and Group D [12]. The detection rate for gastric cancer is highest in the order of group $D, C, B$, and $A$, and thus the $A B C$ method can identify individuals at a higher risk for future gastric cancer development [13-14]. In 2016, a JPHC-based prospective study (Table1) developed a scoring system (the Scoring System from JPHC) for estimating the cumulative probability of gastric cancer occurrence based on a sample of 19,028 individuals, combining age, sex, lifestyle habits (smoking status, consumption of high-sodium food), family history of gastric cancer, as well as biological information [serum anti-H. pylori IgG titers, PG levels] [15]. In recent years, a nationwide multicenter crosssectional study [16] conducted by the China National Clinical Research Center for Digestive Diseases (Shanghai) found that age, sex, pepsinogen I/pepsinogen II ratio (PGR), gastrin-17(G-17), and H. pylori antibodies were significantly associated with the risk of gastric cancer, and developed Li's Scoring System to predict the risk. The study reported that the prediction rule was an accurate, practical, and costeffective prescreening tool for gastric cancer in Chinese high risk populations with good discrimination (AUC of 0.76 ), high sensitivity (70.8\%), and good calibration (0.629), reducing the need for gastroscopies by $66.7 \%$.

However, it is unclear which prediction rule performs best at identifying populations with a higher risk of gastric cancer as candidates for gastroscopies among Chinese health examination populations. Therefore, we aimed to compare the predictive ability of the ABC method, the Scoring System from JPHC, and Li's Scoring System in Chinese health examination populations.

\section{Methods}

\section{Patients}

We retrospectively reviewed patients who had come to the physical examination center for a health examination and undergone upper gastrointestinal tract endoscopy at Songjiang Hospital Affiliated to Shanghai Jiaotong University School of Medicine between March 2019 and September 2020. Serum H. pylori antibody, G-17, and PG I, and PG II data were available. Inclusion criteria: male participants aged 40-79 years and females aged 50-79 years. Exclusion criteria: (1) patients who had received a gastroscopy within 1 year; (2) those with a history of gastrectomy; (3) those treated with a proton pump inhibitor or histamine-2 receptor antagonist within 2 weeks; (4) those with the ischemic anemia within 6 months, gastrointestinal bleeding within 12 months, weight loss, frequent diarrhea, dysphagia or choking,

or an abdominal mass; (5) those with a medical history of esophageal cancer, gastric cancer, colorectal cancer, inflammatory bowel disease, or with other organ malignancies such as breast, ovary, uterus, and 
urinary system malignancies; (6) those highly suspected of having a tumor from image inspections, tumor markers, or other examinations; (7) those with serious mental disorders or serious heart, lung, or renal dysfunction. The study was approved by the Ethics Committee at Songjiang Hospital, Shanghai Jiaotong University School of Medicine (201803), and conformed to the ethical guidelines of the 1975 Helsinki Declaration. Written informed consent was obtained from all subjects.

\section{Serological tests}

Fasting peripheral venous blood samples were collected from eligible subjects during a 10-hour no smoking and no alcohol period to test PGI, PGII, G-17, and H. pylori antibodies. After centrifugation, serum aliquots were stored at $2-8^{\circ} \mathrm{C}$ and immediately assayed within 3 hours. Serum PGI, PGII, G-17, and $H$. pylori antibodies were measured using the Gastro-Panel Mucosal Serological Test Kit (Helicobacter Pylori Antibody Classification Assay Kit: Shenzhen Blot Biotech Co., Ltd, PGI, PGII, and G-17 Test Kit: Biohit Healthcare (Hefei) Co., Ltd).

\section{Gastroscopic and histological examinations}

Gastroscopies were performed by two fixed endoscopists who had performed gastroscopies for more than 10 years, using Olympus GIF-H260Z series electronic gastroscopies (Olympus Corporation, Tokyo, Japan). Patients without obvious lesions underwent a biopsy of the distal greater curvature and distal lesser curvature of the gastric antrum, gastric angulus, and the middle part of the small and large curved sides of the gastric body. Patients with obvious lesions underwent biopsies at those locations. All biopsy specimens were placed on filter paper fixed in a $10 \%$ formalin solution, and sent to the Department of Pathology of our hospital for histopathological diagnosis. Final diagnosis of each histological specimen was independently made by two experienced pathologists.

\section{Treatment and final diagnosis of early cancer cases}

We performed magnifying endoscopies with narrow band imaging (MENBI) and chromoendoscopies on patients diagnosed as indefinite intraepithelial neoplasms, low grade intraepithelial neoplasms (LGIN), and high grade intraepithelial neoplasms (HGIN), using Olympus GIF-H260Z series electronic gastroscopies (Olympus Corporation, Tokyo, Japan). Patients identified as having early cancer underwent endoscopic submucosal dissection (ESD) or surgery. The final diagnoses were based on the pathology of the endoscopy and postoperative pathology. According to the consensus on screening, endoscopic diagnosis and treatment of early gastric cancer in China [11], HGIN was equivalent to severe dysplasia and carcinoma in situ. On the basis of the 2010 Japanese standard, HGIN was equivalent to welldifferentiated gastric tubular adenocarcinomas (tub1) or moderately differentiated tubular adenocarcinoma (tub2). Consequently, we classified HGIN as early cancer.

\section{Statistical analysis}

Continuous variables were presented as the mean with standard deviation, and were compared using Student's $t$-test. Categorical variables were presented as the sample number with corresponding percent, 
and were compared by using the $\chi^{2}$ test. The discrimination of the three prediction rules was assessed by sensitivity, specificity, positive predictive value (PPV), negative predictive value (NPV), ROC curves, and AUC. To compare the AUC, we used DeLong [17]. All statistical analyses were performed using SPSS 19.0, except for the comparison of the paired ROC curves and AUC, for which we used MedCalc 12.0.

\section{Results}

\section{Study populations}

During the study period, 1,436 eligible participants were included in our study, including $362(25.21 \%)$ patients with non-atrophic gastritis, $622(43.31 \%)$ patients with atrophic gastritis, $102(7.10 \%)$ patients with peptic ulcer, 190 (13.23\%) patients with gastric polyps, 10 (0.70\%) patients with LGIN, 28 (1.95\%) patients with gastric cancer, $6(0.42 \%)$ patients with esophageal cancer, and $115(8.01 \%)$ patients with others. Their mean age was $62.70 \pm 10.87$ years, and $65.67 \%$ were men. Among the 28 cases with gastric cancer, $26(92.86 \%)$ cases were early gastric cancer. The patient characteristics are shown in Table 2.

\section{Risk category of the three prediction rules in study populations}

The results with regard to risk category are shown in Table 3 . For the ABC method, $56.48 \%$ of the participants were categorized into the low risk group, whereas $43.45 \%$, and $0.07 \%$ were in the medium risk and high risk groups, respectively. For the Scoring System from JPHC, $76.32 \%$ of the participants were categorized into the low risk group, whereas $18.87 \%$ and $4.81 \%$ were in the medium risk and high risk groups, respectively. For Li's Scoring System, $37.95 \%$ of the participants were categorized into the low risk group, whereas $40.74 \%$ and $21.321 \%$ were in the medium risk and high risk groups, respectively.

\section{Detection of gastric cancer in study populations}

The detection rate of gastric cancer in each risk category of the three prediction rules is shown in Table 3 . The proportion of gastric cancer in low risk groups was highest for the Scoring System from JPHC (1.19\%), followed by the ABC method (0.99\%), and Li's Scoring System (0.55\%). Among the 13 cases of gastric cancer in the low risk group for the Scoring System from JPHC, 11 cases (84.62\%) were 60-79years-old $(60-79$ years vs. $40-59$ years, $p<0.05)$, and the detection rate was similar between men and women. In the low risk group for the ABC method, all cases with gastric cancer were men aged 60-79 years. On the contrary, in the low risk group for Li's Scoring System, all cases with gastric cancer were female. The detection rates of gastric cancer in the medium risk and high risk groups for the $A B C$ method were $3.21 \%$ and 0 , respectively; for the Scoring System from JPHC, they were $2.95 \%$ and $10.14 \%$, respectively; and for Li's Scoring System, they were $1.89 \%$ and $4.58 \%$, respectively. Thus, the detection rate of gastric cancer in the medium risk and high risk groups was significantly higher than that of the low risk group for the three prediction rules $\left(p_{1}<0.05, p_{2}<0.001, p_{3}<0.05\right)$. The detection rate for early gastric cancer in the medium risk and high risk groups was $100 \%$ for both the Scoring System from JPHC and Li's Scoring System, and it was $90 \%$ for the ABC method. 


\section{Performance of the prediction rules}

ROC curves for each prediction rule are depicted in Figure 1. The AUC of the Scoring System from JPHC was highest at $0.745(0.722-0.767)$, followed by Li's Scoring System at $0.739(0.715-0.761)$, and the $A B C$ method, with an AUC of 0.642 (0.617-0.667). The AUC for the Scoring System from JPHC was significantly higher than for the ABC method $(p<0.05)$. No difference was found between the AUC of the Scoring System from JPHC and Li's Scoring System $(p>0.05)$. When categorized as low risk versus higher risk (medium and high risk groups), Li's Scoring System had the highest sensitivity (85.71\%), and the Scoring System from JPHC had the lowest (53.57\%). This difference in sensitivity was statistically significant $(p<0.05)$.All differences in specificity were highly statistically significant (all $p$-values < 0.001). All three prediction rules had high NPV but low PPV (Table 4).

\section{Discussion}

Usually, symptoms of gastric cancer are absent or nonspecific in the early stages of gastric cancer, and they are easily ignored [18]. A prospective study in China involving 102,665 subjects found that $48 \%$ of patients with gastrointestinal malignancy had no indicatory features, and the detection rate of gastrointestinal tumors among those patients was 2.5\% [19]. Thus, such indicatory features alone cannot predict gastrointestinal tumors, and regular endoscopic screening of high-risk patients is key for early diagnosis of gastric cancer. However, the adaptability of the existing risk prediction rules to Chinese health examination populations is not yet clear. Therefore, in this study, we compared the efficacy of three prediction rules: the ABC method, the Scoring System from JPHC, and Li's Scoring System.

In our analysis, the comparison of three prediction rules in 1,436 participants showed that the detection rate of gastric cancer was significantly higher in the medium risk and high risk groups than that in the low risk group $\left(p<0.05, p_{2}<0.001, p_{3}<0.05\right)$ (Table 3$)$, and the detection rate of early gastric cancer in the medium risk and high risk groups reached $90 \%$, indicating that the three prediction rules can improve the detection rate of early gastric cancer. We noticed that all cases of advanced gastric cancer were diffusetype lesions, which were detected in the low risk group for both the Scoring System from JPHC and Li's Scoring System. Previous studies reported that these scoring systems did not perform well for diffusetype gastric cancer [15-16].

One valuable role of prediction rules is to identify patients as low risk in order to avoid further testing and save medical resources. An ideal risk prediction rule would identify low risk patients of considerable size with a low detection rate of gastric cancer, to minimize missed diagnoses. However, it is worth noting that a number of studies mentioned that gastric cancer and precancerous lesions were detected in the low risk groups of several prediction rules. [16,20-21]. In our study, the Scoring System from JPHC identified the most patients as low risk (1096, 76.32\%), followed by the ABC method $(811,56.48 \%)$, and Li's Scoring System (37, 37.95\%). The missed diagnosis rate of gastric cancer was the highest in the Scoring System from JPHC (1.19\%), and $84.62 \%$ of the patients aged 60-79 years. Therefore, missed diagnoses should be especially considered for elderly patients when using this scoring system. The missed diagnosis rate 
of the ABC method was $0.99 \%$, and all cases were males, which should be taken seriously. Furthermore, patients in group A with a history of $\mathrm{H}$. pylori eradication may need gastroscopy. Miura et al. reported that about $20 \%$ patients with gastric cancer have a history of $H$. pylori eradication, and they have normal PG levels and negative anti-H. pylori lgG titres [22]. Although Li's Scoring System had the lowest rate of missed diagnosis $(0.55 \%)$, all missed patients were females. Thus, it is still necessary to consider the missed diagnosis of female patients due to the assigned 0 points for females in Li's scoring system.

In this study, the ABC method showed the least predictive capacity for gastric cancer with the lowest AUC (0.642, 95\% Cl: 0.617-0.667), which was significantly lower than that of the Scoring System from JPHC $(p<0.05)$. Moreover, the specificity was significantly lower than that of the Scoring System from JPHC ( $p$ $<0.001)$ and Li's Scoring System $(p<0.001)$. Consistent with previous research, the ABC method for gastric cancer screening did not work well in the Chinese populations, with lower sensitivity and specificity than those in nonChinese studies [23-24]. This may be related to the fact that the ABC method was developed in Japanese populations, and the optimal PG and G-17 cut-off values may not be applicable to screening for gastric cancer in China. Furthermore, the $A B C$ method only includes the results of serological indicators without demographic factors (e.g. age, sex, etc.) and lifestyle habits (e.g. smoking status, consumption of alcohol and high-sodium food, etc.), which may affect its predictive ability.

The Scoring System from JPHC had the highest AUC (0.745, 95\% Cl: $0.722-0.767)$, followed by Li's Scoring System $(0.739,95 \% \mathrm{Cl}: 0.715-0.761)$, and these two prediction rules performed similarly. However, the Scoring System from JPHC had the largest possibility of missed diagnosis of gastric cancer with the lowest sensitivity (53.57\%) and NPV(98.90\%) $\$ which may be possibly explained by the weight setting of indicators (e.g. H. pylori infection) and regional differences. The Scoring System from JPHC was developed based on a large group of Japanese participants and included a number of variables, such as demographic factors, lifestyle, and the risk categories of the $A B C$ method. Furthermore, the optimal cut-off values of PG and G-17 may be inappropriate for screening in China [15]. The scoring system did not find any substantial difference between the risk of individuals in categories $C$ and $D$ of the ABC method, which were similar to several published studies [25-26]. Nevertheless, the scoring system emphasized the important effect of smoking and high sodium food consumption on the occurrence of gastric cancer, and the quantification of these lifestyle-related risk factors might provide an incentive for adopting healthier lifestyles, particularly for high-risk individuals [27].

Li's Scoring System performed well in predicting the risk of gastric cancer and having the lowest probability of missed diagnosis with the modest AUC (0.739), and highest sensitivity (85.71\%) and NPV (99.50\%). These results are similar to the originally published values [16]. The prediction rule was developed based on a multicenter, large-sample Chinese population, and the cut-off values of PG and G17 were more in line with screening for gastric cancer in China. For example, the cut-off value of PGR in Li's Scoring System and the Scoring System from JPHC is 3.89 and 3, respectively. However, it is worth noting that the efficacy of Li's Scoring System should be further verified in order to improve discrimination and reduce missed diagnosis of females. H. pylori infection has been considered as the key cause of 
intestinal gastric cancer, and the efficacy of screening may be improved by distinguishing two types of $H$. pylori strains (strains expressing CagA and VacA, and strains without CagA and VacA).

Our study had potential limitations. First, we studied the ABC method, the Scoring System from JPHC, and Li's Scoring System, although several other prediction rules are available $[28,17]$. Second, we only compared the efficacy of three prediction rules, and did not analyze possible risk factors (e.g., obesity and the consumption of fried food, etc.), which could be done by gathering more cases in future studies. Finally, the sample size was small. We only included 1436 health examination populations, suggesting Chinese are poorly informed about gastric cancer screening and the education on this topic is needed.

\section{Conclusion}

In conclusion, we report that Li's Scoring System is more useful in Chinese health examination populations because of its lowest possibility of missed diagnoses of gastric cancer, though no significant differences are found between the Scoring System from JPHC and Li's Scoring System in predicting the risk of gastric cancer. However, missed diagnoses among female patients for Li's Scoring System cannot be ignored, and future studies are needed to validate its efficacy and to explore a screening process that is more practical in China.

\section{Abbreviations}

Japan Public Health Center: JPHC; areas under the receiver-operating characteristic: ROC; areas under the receiver-operating characteristic curves: AUC; Helicobacter pylori. H. pylori; pepsinogen: PG; pepsinogen I/pepsinogen II ratio: PGR; gastrin-17: G-17; magnifying endoscopies with narrow band imaging: MENBl; low grade intraepithelial neoplasms: LGIN; high grade intraepithelial neoplasms: HGIN; endoscopic submucosal dissection: ESD; well-differentiated gastric tubular adenocarcinomas: tub1; moderately differentiated tubular adenocarcinoma: tub2; positive predictive value: PPV; negative predictive value: NPV.

\section{Declarations}

\section{Ethics approval and consent to participate}

The study has been approved by the Ethics Committee at Songjiang Hospital, Shanghai Jiaotong University School of Medicine (201803), and conformed to the ethical guidelines of the 1975 Helsinki Declaration. Written informed consent was obtained from all subjects.

\section{Consent for publication}

All named authors have agreed to the paper publication.

\section{Availability of data and materials}


Currently, the data are not yet openly available. The study group welcomes potential collaboration to maximize use of existing resources. The datasets analysed in this study are available from the corresponding author on reasonable request.

\section{Competing interests}

The authors have declared no conflicts of interest.

\section{Funding}

The National Natural Science Foundation of China (81702317) and the Scientific Research Projects of the Shanghai Municipal Health Commission (201840229) provide financial support during questionnaire distribution and data collection.

\section{Authors' contributions}

PX and YL designed the study. All the authors performed sample collection and data analysis.

$\mathrm{XX}$ wrote the initial manuscript under the guidance of $\mathrm{YL}$. $\mathrm{XX}, \mathrm{YL}, \mathrm{PX}$, and JW contributed to the data interpretation and manuscript revision. All the authors have read the manuscript, PX, YL and JW approved the final manuscript.

\section{Acknowledgements}

We thank International Science Editing (http://www.internationalscienceediting.com) for editing this manuscript.

\section{References}

1. Chen W, Zheng R, Baade PD, Zhang S, Zeng H, Bray F, et al. Cancer statistics in China, 2015. CA Cancer J Clin. 2016; 66(2):115-32.

2. Oliveira C, Pinheiro H, Figueiredo J, Seruca R, Carneiro F. Familial gastric cancer: genetic susceptibility, pathology, and implications for management. Lancet Oncol. 2015;16(2):e60-70.

3. Zhang X, Li M, Chen S, Hu J, Guo Q, Liu R, et al. Endoscopic Screening in Asian Countries Is Associated With Reduced Gastric Cancer Mortality: A Meta-analysis and Systematic Review. Gastroenterology. 2018;155(2):347-54.

4. Katai H, Ishikawa T, Akazawa K, Isobe Y, Miyashiro I, Oda I, et al. Five-year survival analysis of surgically resected gastric cancer cases in Japan: a retrospective analysis of more than 100,000 patients from the nationwide registry of the Japanese Gastric Cancer Association (2001-2007). Gastric Cancer. 2018;21(1):144-54.

5. Banks M, Graham D, Jansen M, Gotoda T, Coda S, di Pietro M, et al. British Society of Gastroenterology guidelines on the diagnosis and management of patients at risk of gastric adenocarcinoma. Gut. 2019;68(9):1545-75. 
6. Hamashima C, Goto R. Potential capacity of endoscopic screening for gastric cancer in Japan. Cancer Sci. 2017;108(1):101-7.

7. Lin JT. Screening of gastric cancer: who, when, and how. Clin Gastroenterol Hepatol. 2014;12(1):1358.

8. Chen R, Liu Y, Song G, Li B, Zhao D, Hua Z, et al. Effectiveness of one-time endoscopic screening programme in prevention of upper gastrointestinal cancer in China: a multicentre population-based cohort study. Gut. 2021; 70 (2):251-60.

9. Jun JK, Choi KS, Lee HY, Suh M, Park B, Song SH, et al. Effectiveness of the Korean National Cancer Screening Program in Reducing Gastric Cancer Mortality. Gastroenterology. 2017;152(6):1319-28.

10. Zhu L, Qin J, Wang J, Guo T, Wang Z, Yang J. Early Gastric Cancer: Current Advances of Endoscopic Diagnosis and Treatment. Gastroenterol Res Pract. 2016;2016:9638041.

11. Chinese Society of Digestive Endoscopy. Consensus on screening and endoscopic diagnosis and treatment of early gastric cancer in China (Changsha, 2014). Zhonghua Xiao Hua Nei Jing Za Zhi. 2014;31(7):361-77.

12. Miki K. Gastric cancer screening by combined assay for serum anti-Helicobacter pylori lgG antibody and serum pepsinogen levels - "ABC method". Proc Jpn Acad Ser B Phys Biol Sci. 2011;87(7):405-14.

13. Ikeda F, Shikata K, Hata J, Fukuhara M, Hirakawa Y, Ohara T, et al. Combination of Helicobacter pylori Antibody and Serum Pepsinogen as a Good Predictive Tool of Gastric Cancer Incidence: 20-Year Prospective Data From the Hisayama Study. J Epidemiol. 2016;26(12):629-36.

14. Sasazuki, Shizuka. The ABC method and gastric cancer: Evidence from prospective studies. Journal of Epidemiology. 2016;26(12):611-2.

15. Charvat H, Sasazuki S, Inoue M, Iwasaki M, Sawada N, Shimazu T, et al. Prediction of the 10-year probability of gastric cancer occurrence in the Japanese population: the JPHC study cohort II. Int J Cancer. 2016;138(2):320-31.

16. Cai Q, Zhu C, Yuan Y, Feng Q, Feng Y, Hao Y, et al. Development and validation of a prediction rule for estimating gastric cancer risk in the Chinese high-risk population: a nationwide multicentre study. Gut. 2019;68(9):1576-87.

17. Carlton EW, Khattab A, Greaves K. Identifying patients suitable for discharge after a singlepresentation high-sensitivity troponin result: A comparison of five established risk scores and two high-sensitivity assays. Ann Emerg Med. 2015;66(6):635-45.

18. Choi IJ. Endoscopic gastric cancer screening and surveillance in high-risk groups. Clin Endos 2014;47(6):497-503.

19. Bai Y, Li ZS, Zou DW, Wu RP, Yao YZ, Jin ZD, et al. Alarm features and age for predicting upper gastrointestinal malignancy in Chinese patients with dyspepsia with high background prevalence of Helicobacter pylori infection and upper gastrointestinal malignancy: an endoscopic database review of 102,665 patients from 1996 to 2006. Gut. 2010;59(6):722-8.

20. Kishikawa H, Kimura K, Ito A, Arahata K, Takarabe S, Kaida S, et al. Cutoff Pepsinogen Level for Predicting Unintendedly Eradicated Cases of Helicobacter pylori Infection in Subjects with Seemingly 
Normal Pepsinogen Levels. Digestion. 2017;95(3):229-236.

21. Kishino T, Oyama T, Tomori A, Takahashi A, Shinohara T. Usefulness and Limitations of a Serum Screening System to Predict the Risk of Gastric Cancer. Intern Med. 2020; 59(12):1473-80.

22. Miura K, Okada H, Kouno Y, Kanzaki H, Iwamuro M, Hori K, et al. Actual Status of Involvement of Helicobacter pylori Infection That Developed Gastric Cancer from Group A of ABC (D) Stratification Study of Early Gastric Cancer Cases That Underwent Endoscopic Submucosal Dissection. Digestion. 2016;94(1):17-23.

23. Tu H, Sun L, Dong X, Gong Y, Xu Q, Jing J, et al. A Serological Biopsy Using Five Stomach-Specific Circulating Biomarkers for Gastric Cancer Risk Assessment: A Multi-Phase Study. Am J Gastroenterol. 2017;112(5):704-15.

24. Yu G, Wang GX, Wang HG, Mo FF, Tang BB. The value of detecting pepsinogen and gastrin-17 levels in serum for pre-cancerous lesion screening in gastric cancer. Neoplasma. 2019;66(4):637-40.

25. Ikeda F, Shikata K, Hata J, Fukuhara M, Hirakawa Y, Ohara T, et al. Combination of Helicobacter pylori Antibody and Serum Pepsinogen as a Good Predictive Tool of Gastric Cancer Incidence: 20-Year Prospective Data From the Hisayama Study. J Epidemiol. 2016; 26(12):629-36.

26. Terasawa T, Nishida H, Kato K, Miyashiro I, Yoshikawa T, Takaku R, et al. Prediction of gastric cancer development by serum pepsinogen test and Helicobacter pylori seropositivity in Eastern Asians: a systematic review and meta-analysis. PLoS One. 2014;9(10):e109783.

27. Liu Q, Zeng X, Wang W, Huang RL, Huang YJ, Liu S, et al. Awareness of risk factors and warning symptoms and attitude towards gastric cancer screening among the general public in China: a crosssectional study. BMJ Open. 2019; 9(7):e029638.

28. Tu H, Sun L, Dong X, Gong Y, Xu Q, Jing J, et al. A Serological Biopsy Using Five Stomach-Specific Circulating Biomarkers for Gastric Cancer Risk Assessment: A Multi-Phase Study. Am J Gastroenterol. 2017; 112(5):704-15.

\section{Tables}

Table 1 Factors in prediction rules 


\begin{tabular}{|c|c|}
\hline Prediction rule & Specific details \\
\hline \multicolumn{2}{|l|}{ ABC method } \\
\hline PG(-), Anti-H. pylori lgG (-) & Group A \\
\hline PG(-), Anti-H. pylori lgG (+) & Group B \\
\hline PG(+), Anti-H. pylori lgG (+) & Group C \\
\hline PG(+), Anti-H. pylori lgG (-) & Group D \\
\hline Li's Scoring System & $0-23$ points \\
\hline \multicolumn{2}{|l|}{ Age } \\
\hline $40-49$ & 0 points \\
\hline $50-59$ & 5 points \\
\hline $60-69$ & 6 points \\
\hline$>69$ & 10 points \\
\hline \multicolumn{2}{|l|}{ Sex } \\
\hline Female & 0 points \\
\hline Male & 4 points \\
\hline \multicolumn{2}{|l|}{ H. pylori antibody } \\
\hline Negative & 0 points \\
\hline Positive & 1 points \\
\hline \multicolumn{2}{|l|}{ PGR } \\
\hline$\geq 3.89$ & 0 points \\
\hline$<3.89$ & 3 points \\
\hline \multicolumn{2}{|l|}{ G-17 } \\
\hline$\leq 1.49$ & 0 points \\
\hline $1.5-5.7$ & 3 points \\
\hline$>5.7$ & 5 points \\
\hline Scoring System from JPHC & $0-24$ points \\
\hline Age & \\
\hline
\end{tabular}

Page 12/17 


\begin{tabular}{|ll|}
\hline $40-44$ female/male & $0 / 1$ points \\
\hline $50-59$ female/male & $1 / 3$ points \\
\hline $55-59$ female/male & $2 / 4$ points \\
\hline $60-64$ female/male & $3 / 6$ points \\
\hline $65-71$ female/male & $4 / 8$ points \\
\hline Smoking status & $5 / 10$ points \\
\hline Never, past & \\
\hline Current & 0 points \\
\hline Salted roe & 1 points \\
\hline No & 0 points \\
\hline yes & 1 points \\
\hline Family history of gastric cancer & \\
\hline No & 0 points \\
\hline yes & 1 points \\
\hline ABC risk category & 11 points \\
\hline A & 0 points \\
\hline B & 8 points \\
\hline C & 11 points \\
\hline D & \\
\hline
\end{tabular}

PG, pepsinogen; G-17, gastrin-17; H. pylori, Helicobacter pylori; JPHC, Japan Public Health Center.

The $A B C$ method designates three risk categories, with patients defined as low risk if they are classified in Group A, medium risk if they are classified in Groups B and C, and high risk if they are classified in Group D.

The Scoring System from JPHC designates three risk categories, with patients defined as low risk if the score is $\leq 14$, medium risk if the score is $15-19$, and high risk if the score is $\geq 20$.

Li's Scoring System designates three risk categories, with patients defined as low risk if the score is $\leq 11$, medium risk if the score is $12-16$, and high risk if the score is $\geq 17$. 
Table 2 Baseline and clinical characteristics

\begin{tabular}{|ll|}
\hline Characteristics & Mean \pm SD, or $n(\%)$ \\
\hline Age & $62.70 \pm 10.87$ \\
\hline Sex (male) & $943(65.67 \%)$ \\
\hline PGI (ng/L) & $134.52 \pm 61.78$ \\
\hline PGI (ng/L) & $9.59 \pm 6.26$ \\
\hline G-17 (pmol/L) & $17.54 \pm 10.87$ \\
\hline H. pylori antibody (positive) & $4.75 \pm 6.42$ \\
\hline Smoking & $624(43.45 \%)$ \\
\hline Salty food & $481(33.50 \%)$ \\
\hline Family history of gastric cancer & $1046(72.84 \%)$ \\
\hline Endoscopic diagnosis & $59.11 \%)$ \\
\hline Non-atrophic gastritis & $362(25.21 \%)$ \\
\hline Atrophic gastritis & $622(43.31 \%)$ \\
\hline Peptic ulcer & $102(7.10 \%)$ \\
\hline Gastric polyps & $190(13.23 \%)$ \\
\hline LGIN & $10(0.70 \%)$ \\
\hline Gastric cancer & $28(1.95 \%)$ \\
\hline Early gastric cancer & $26(92.86 \%)$ \\
\hline tub1 & $20(76.92 \%)$ \\
\hline tub1 > tub2 & $2(7.69 \%)$ \\
\hline tub2 & $3(11.54 \%)$ \\
\hline muc & $1(3.85 \%)$ \\
\hline Advanced gastric cancer & $2(7.14 \%)$ \\
\hline por & $2(100 \%)$ \\
\hline Esophageal cancer & $115(8.42 \%)$ \\
\hline Others & \\
\hline
\end{tabular}


PG, pepsinogen; PGR, pepsinogen I/pepsinogen II; G-17, gastrin-17; H. pylori, Helicobacter pylori, LGIN, low grade intraepithelial neoplasia.

Table 3 Detection rate of gastric cancer in study populations

\begin{tabular}{|c|c|c|c|c|c|c|c|c|c|}
\hline \multirow{3}{*}{$\begin{array}{l}\text { Prediction } \\
\text { rule }\end{array}$} & \multirow{3}{*}{$\begin{array}{l}\text { Risk } \\
\text { category }\end{array}$} & \multirow[t]{3}{*}{$N, \%$} & \multicolumn{7}{|c|}{ Detection rate of gastric cancer, $\mathrm{N}(\%)$} \\
\hline & & & \multicolumn{4}{|l|}{ Age } & \multicolumn{2}{|l|}{ Sex } & \multirow[t]{2}{*}{ Total } \\
\hline & & & $\begin{array}{l}40- \\
49\end{array}$ & $\begin{array}{l}50- \\
59\end{array}$ & $\begin{array}{l}60- \\
69\end{array}$ & $70-79$ & Male & Female & \\
\hline \multirow{6}{*}{$\begin{array}{l}\text { ABC } \\
\text { method }^{1}\end{array}$} & Low & 811 & 0 & 0 & 2 & 6 & 8 & 0 & 8 \\
\hline & & $(56.48)$ & & & $(0.87)$ & $(1.82)$ & $(1.48)$ & & $(0.99)$ \\
\hline & Medium & 624 & 2 & 0 & 7 & 11 & 15 & 5 & 20 \\
\hline & & $(43.45)$ & $(1.92)$ & & $(3.76)$ & $(5.91)$ & $(3.73)$ & $(2.25)$ & $(3.21)$ \\
\hline & High & 1 & 0 & 0 & 0 & 0 & 0 & 0 & 0 \\
\hline & & $(0.07)$ & & & & & & & \\
\hline \multirow{6}{*}{$\begin{array}{l}\text { Scoring } \\
\text { System } \\
\text { from } \\
\text { JPHC }^{2}\end{array}$} & Low $^{4}$ & 1096 & 2 & 0 & 4 & 7 & 8 & 5 & 13 \\
\hline & & (76.32) & $(0.87)$ & & $(1.60)$ & (1.92) & $(1.32)$ & $(1.02)$ & $(1.19)$ \\
\hline & Medium & 271 & 0 & 0 & 4 & 4 & 8 & 0 & 8 \\
\hline & & (18.87) & & & (3.28) & (3.88) & $(2.97)$ & & $(2.95)$ \\
\hline & High & 69 & 0 & 0 & 1 & 6 & 7 & 0 & 7 \\
\hline & & $(4.81)$ & & & $(4.76)$ & (12.77) & $(10.29)$ & & (10.14) \\
\hline \multirow{6}{*}{$\begin{array}{l}\text { Li's } \\
\text { Scoring } \\
\text { System }^{3}\end{array}$} & Low & 545 & 2 & 0 & 1 & 0 & 0 & 3 & 3 \\
\hline & & (37.95) & $(0.87)$ & & $(0.70)$ & & & $(0.78)$ & $(0.55)$ \\
\hline & Medium & 585 & 0 & 0 & 8 & 3 & 9 & 2 & 11 \\
\hline & & (40.74) & & & (3.23) & $(1.48)$ & $(1.88)$ & $(1.89)$ & $(1.89)$ \\
\hline & High & 306 & 0 & 0 & 0 & 14 & 14 & 0 & 14 \\
\hline & & (21.31) & & & & $(4.62)$ & $(4.61)$ & & $(4.58)$ \\
\hline
\end{tabular}

JPHC, Japan Public Health Center.

$1,2,3$, the detection rate of gastric cancer of the high risk group was significantly higher than that of the low risk group in three prediction rules $\left(p_{1}<0.05, p_{2}<0.001, p_{3}<0.05\right)$. 
4 , In the low risk group for the Scoring System from JPHC, the detection rate of gastric cancer of patients aged 60-79 years was significantly higher than that of those aged $50-59$ years $(p<0.05)$.

Table 4 Performance of the prediction rules in the study populations

\begin{tabular}{|llll|}
\hline & ABC method & Scoring System from JPHC & Li's Scoring System \\
\hline Sensitivity $^{\mathrm{a}}$ & $71.40 \%$ & $53.57 \%$ & $85.71 \%$ \\
\hline Specificity & $57.00 \%$ & $81.39 \%$ & $56.75 \%$ \\
\hline PPV & $3.20 \%$ & $5.40 \%$ & $3.80 \%$ \\
\hline NPV & $99.0 \%$ & $98.90 \%$ & $99.50 \%$ \\
\hline AUC & $0.642(0.617-0.667)$ & $0.745(0.722-0.767)$ & $0.739(0.715-0.761)$ \\
\hline
\end{tabular}

PPV, positive predictive value; NPV, negative predictive value; AUC, area under the curve; JPHC, Japan Public Health Center.

${ }^{a}$ The sensitivity of Li's Scoring System was significantly higher than of the Scoring System from JPHC ( $p$ $<0.05)$

${ }^{\mathrm{b}}$ All differences in specificity were highly statistically significant (all $p$-values $<0.001$ ).

${ }^{\mathrm{C}}$ The AUC for the Scoring System from JPHC was significantly higher than for the ABC method $(p<0.05)$.

\section{Figures}




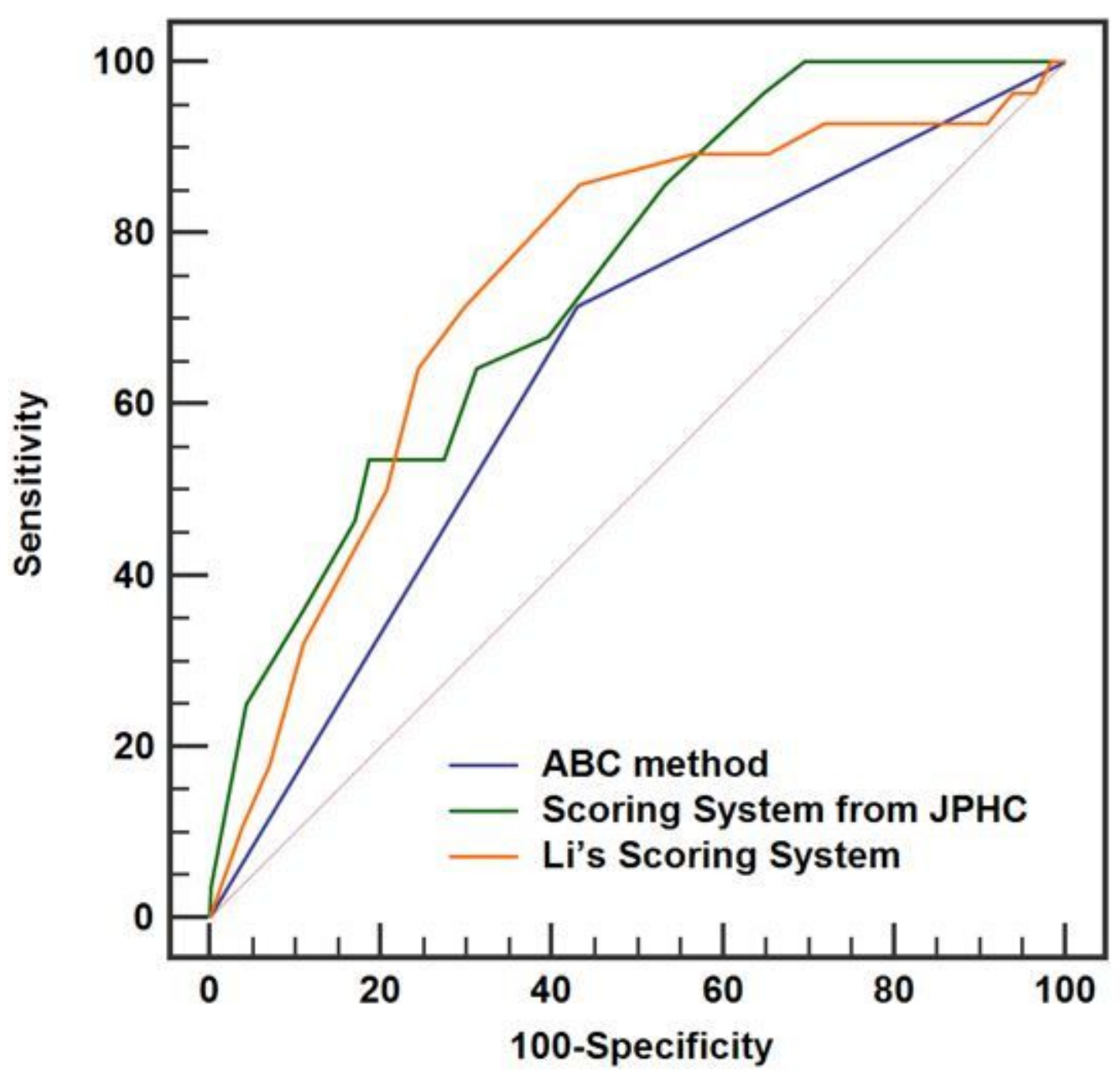

Figure 1

Receiver operating characteristic curves for the ABC method, the Scoring System from JPHC, and Li's Scoring System in the study populations. 